\title{
Renal AA-amyloidosis in intravenous drug users - a role for HIV-infection?
}

\author{
Oliver Jung ${ }^{1}$, Hans Stefan Haack², Maike Buettner ${ }^{3}$, Christoph Betz ${ }^{1}$, Christoph Stephan ${ }^{4}$, Peter Gruetzmacher ${ }^{2}$, \\ Kerstin Amann ${ }^{3}$ and Markus Bickel ${ }^{4,5^{*}}$
}

\begin{abstract}
Background: Chronic renal disease is a serious complication of long-term intravenous drug use (IVDU). Recent reports have postulated a changing pattern of underlying nephropathy over the last decades.

Methods: Retrospective investigation including all patients with prior or present IVDU that underwent renal biopsy because of chronic kidney disease between 01.04.2002 and 31.03.2012 in the city of Frankfurt/Main, Germany.

Results: Twenty four patients with IVDU underwent renal biopsy because of progressive chronic kidney disease or proteinuria. Renal AA-amyloidosis was the predominant cause of renal failure in $50 \%$ of patients.

Membranoproliferative glomerulonephritis (GN) was the second most common cause found in $21 \%$. Patients with AA-amyloidosis were more likely to be HIV infected (67 vs.17\%; $p=0.036$ ) and tended to have a higher rate of repeated systemic infections ( 92 vs. 50\%; $\mathrm{p}=0.069$ ). Patients with $\mathrm{AA}$-amyloidosis presented with progressive renal disease and nephrotic-range proteinuria but most patients had no peripheral edema or systemic hypertension. Development of proteinuria preceded the decline of GFR for approximately 1-2 years.

Conclusions: AA-amyloidosis was the predominant cause of progressive renal disease in the last 10 years in patients with IVDU. The highest rate of AA-amyloidosis observed was seen in HIV infected patients with IVDU. We speculate that chronic HIV-infection as well as the associated immunosuppression might promote development of AA-amyloidosis by increasing frequency and duration of infections acquired by IVDU.
\end{abstract}

Keywords: AA-amylodosis, IVDU, Chronic kidney disease, HIV

\section{Background}

Renal disease related to intravenous drug use (IVDU) has been reported since the 1970 s, mostly in the context of heroin-associated nephropathy, characterized by nephritic syndrome and rapid-progression [1,2]. The incidence of heroin associated-nephropathy decreased in the last four decades. More recent reports starting from the 1990s related chronic kidney disease in IVDU more with concomitant chronic HIV-, HBV- and HCVinfection [3-5]. Two recent studies from Europe observed changing patterns of renal disease in patients

\footnotetext{
* Correspondence: markus.bickel@hivcenter.de

${ }^{4}$ Department of Infectious Disease, Goethe University, Frankfurt/Main, Germany

${ }^{5}$ Klinikum der Goethe-Universität, Zentrum der Inneren Medizin II, Infektionsambulanz, Haus 68, 1 OG, Theodor Stern Kai 7, 60590, Frankfurt/Main, Germany

Full list of author information is available at the end of the article
}

with IVDU, reporting an increased prevalence of renal AA-amyloidosis [6,7].

Renal AA-amyloidosis is a complication of chronic and/or recurrent inflammatory disease [8]. Autoimmune diseases, mainly rheumatoid arthritis, are common causes in resource rich countries whereas untreated chronic infections are the predominant cause in countries with limited medical resources. Severe proteinuria, nephrotic syndrome as well as renal insufficiency are the typical clinical manifestations of renal AA-amyloidosis [8]. Successful treatment of the underlying inflammation, by immunosuppressant's for autoimmune diseases or by antimicrobials for chronic infections, can lead to stabilization of or even improvements of renal function [9].

Although cases of AA-amyloidosis in patients with IVDU have already been described in the 1970s [10,11], only sporadic cases have been reported in the last decades [5,12-14]. To date, it remains unclear to what

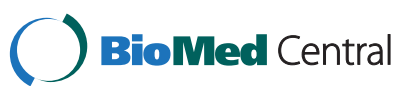


extent IVDU and associated co-morbidities, especially chronic infections, contribute to the development of renal AA-amyloidosis. Our aim was to describe the histologic pattern of nephropathy and its associated risk factors in a contemporary cohort of patients with IVDU.

\section{Methods}

The Nephrology departments at Goethe-University (GU) and the Agaplesion Markus Krankenhaus (AMK) are two main nephrologic care providers and the only centres conducting renal biopsies in Frankfurt, Germany. All patients with IVDU were identified by using the Patient Data management System of the renal units of the GU and AMK that are based on primary and secondary diagnosis according to the international Classification of Diseases 10th Revision (ICD-10). We then retrospectively analyzed all patients with ongoing or a prior history of IVDU referred to us between 01.04.2002 and 31.03.2012 that underwent renal biopsy because of chronic kidney disease. Biopsies were performed because of progressive renal failure or overt proteinuria. Clinical data and the patient/s medical history were extracted from the medical records. Glomerular filtration rate was estimated (eGFR) using the Modification of Diet in Renal Disease (MDRD) equation [15]. Proteinuria was assessed by collection of 24-h urine at the time of renal biopsy. Proteinuria prior to renal biopsy was determined from spot urine. End-stage renal disease (ESRD) was defined as initiation of chronic renal replacement therapy (RRT) in an ambulatory setting. All data were collected retrospectively; thus no informed consent or approval from the ethics committee was obtained.

Chronic hepatitis B virus (HBV) infection was defined by a detectable HBs antigen persisting at least 6 months. Past HBV exposure was defined by detection of HBV core or surface antibodies in absence of previous vaccination. Chronic hepatitis $\mathrm{C}$ virus (HCV) infection was defined by detection of $\mathrm{HCV}$ antibodies and positive HCV RNA PCR persisting at least 6 months. HIV infection was defined by positive HIV antibodies or positive HIV RNA PCR.

All renal biopsy specimens were submitted for diagnostic purposes and proceeded using our standardized routine protocol. Briefly, formalin-fixed and paraffinembedded kidney biopsy specimens were sectioned into $2 \mu \mathrm{m}$ thick paraffin sections with at least 8 serial sections stained either by periodic acid-Schiff (PAS) or hematoxylin-eosin (HE) stainings. Additionally, a Congo red staining as well as immunohistochemical stainings with antisera specific for IgA (1:150000), IgG (1:100000), IgM (1:75000), C1q (1:75000) and C3c (1:75000, all polyclonal rabbit antisera, Dako Cytomation, Hamburg, Germany) were performed of all cases. In Congo red positive cases additional stainings with antibodies specific for the kappa (1:50000) and lambda (1:100000) light chains (both polyclonal rabbit antisera, Dako) and for amyloid A (1:500, monoclonal, mouse, clone mc-1, Dako) for differentiation of the amyloid deposits were initiated.

Continuous variables were expressed as median and interquartile-range (IQR) or as proportions as appropriate. Continuous and categorical variables were compared for univariate analysis between groups using the $t$-test or Mann-Whitney $U$-test and Fisher exact test, respectively. All p-values reported are two-sided. Statistical significance was assumed when the $\mathrm{p}$-value was $<0.05$. A statistical trend was assumed when the $\mathrm{p}$-value was $>0.05$ but $<0.08$. Kaplan-Meier (Mantel-Cox log-rank test).estimates were used to analyze time to death or progression to ESRD.

\section{Results}

Patients characteristics and renal findings on biopsy Between 01.04.2002 and 31.03.2012 a total of 27 IVDU patients were referred to our units because of chronic kidney disease with either progressive renal failure or large proteinuria. Renal biopsy was performed in 24 $(88.9 \%)$ of these patients. A renal biopsy was not performed in one patient because of impaired coagulation due to liver cirrhosis and two patients declined renal biopsy. Patients were followed after renal biopsy for a cumulative observation time of 59.5 patient-years (range 4 to 3540 days). All patients were Caucasians and mostly male (62.5\%). Self reported duration of IVDU was 333 years and 12 patients (50\%) had been, at least intermittently, on opioid maintenance therapy. Chronic viral infections were frequent: 21 patients $(87.5 \%)$ had a chronic HCV infection, 10 patients (41.7\%) an HIV infection and one (4.2\%) had chronic HBV infection. Seven patients $(29.2 \%)$ had serologic findings indicating prior HBV exposure.

Twelve patients (50\%) had renal AA-amyloidosis and five patients (20.8\%) had a membranoproliferative glomerulonephritis type I. All other types of renal disease were seen only in 1 or 2 patients (Table 1 ).

\section{Clinical characteristics of patients with renal AA-amyloidosis}

All 12 patients with renal AA-amyloidosis presented with elevated serum creatinine and nephrotic-range proteinuria, but only 3 (25\%) had signs of peripheral edema in physical examination. Hypertension was uncommon and found only in one patient (8.3\%). Ultrasound examination revealed enlarged kidneys with increased echogenicity in all patients. All patients had a medical history of chronic or repeated infections. Examination of renal biopsies showed deposition of AA-amyloid in the 
Table 1 Patients characteristics and renal findings on biopsy

\begin{tabular}{lc}
\hline Age (years) & $41(33-46)$ \\
\hline Male $-\mathrm{n}(\%)$ & $15(62.5 \%)$ \\
\hline Self-reported time after initiation of IVDU (years) & $22(13-30)$ \\
\hline Self-reported duration of IVDU in years & $17(8-25)$ \\
\hline History of opioid maintenance therapy & $12(50 \%)$ \\
\hline Chronic hepatitis B & $1(4.2 \%)$ \\
\hline Past HBV exposure & $7(29.2 \%)$ \\
\hline Chronic hepatitis C & $21(87.5 \%)$ \\
\hline HIV infection & $10(41.7 \%)$ \\
\hline Renal disease by biopsy-findings - n (\%) & $12(50.0 \%)$ \\
\hline AA-amyloidosis & $5(20.8)$ \\
\hline Membranoproliferative GN Type I & $2(8.3 \%)$ \\
\hline Nephrosclerosis & $2(8.3 \%)$ \\
\hline Extracapillary proliferative GN & $1(4.2 \%)$ \\
\hline Focal segmental Glomerulosclerosis & $1(4.2 \%)$ \\
\hline Chronic tubulointerstitial nephritis & $1(4.2 \%)$ \\
\hline Lupus nephritis Class IV
\end{tabular}

Variables are expressed as median and interquartile-range (IQR) or as proportions as appropriate.

glomerula, the vessels and the interstitial compartment. Tubular atrophy and interstitial fibrosis was present in all patients ranging from 20 to $80 \%$ (representative histological findings are presented in Figure 1). Two patients had additional signs of acute interstitial nephritis.

\section{Time course of renal AA-amyloidosis}

For 10 out of 12 patients with AA-amyloidosis we were able to retrieve medical records on kidney function prior to the time of renal biopsy. All patients showed a transient period of hyperfiltration followed by a rapid decline of the eGFR within less than 2 years. Proteinuria preceded this eGFR decline a median of 20 months (range 13-36 months). Clinical course of renal AA-amyloidosis prior to biopsy is demonstrated in Figure 2.

Two patients died within 1 year after diagnosis (one because of sepsis, one of acute drug intoxication), and five additional patients progressed to ESRD within less than 2 years. Among those five patients with ESRD, three patients died within 2 years after initiation of renal replacement therapy (one because of sepsis, one of drug intoxication, one by suicide). Among patients with other causes of renal disease, 3 patients progressed to ESRD. Outcome of patients with established diagnosis of renal AA-amyloidosis is shown in Figure 3, demonstrating the poorer outcome of these patients $(\mathrm{p}=0.0782)$.

Four surviving patients did not progress to ESRD within more than 2 years after the diagnosis; all had stopped IVDU after diagnosis of AA-amyloidosis and were successfully treated with opioid maintenance therapy.

\section{Comparison of patients characteristics between those with or without renal AA-amyloidosis}

Both groups of patients were comparable for most demographic and clinical characteristics (Table 2). Amount of proteinuria was higher in patients with renal AA-amyloidosis as compared to those with other types of renal disease $(p=0.021)$. HIV infection was significantly more frequent in patients with AA-amyloidosis than in those with other types of chronic renal disease (67 vs. $17 \% ; \mathrm{p}=0.036$ ). Patients with $\mathrm{AA}$-amyloidosis tended to have a longer self-reported duration of IVDU (20.5 vs. 12.5 months; $\mathrm{p}=0.056)$ and a higher frequency of chronic or repeated infections ( 92 vs $50 \%$; $\mathrm{p}=0.069$ ) (Table 2). Of the eight patients with HIV infection and renal AA-amyloidosis, three patients had previous AIDS-defining events and four patients were previously naive to antiretroviral therapy at the time of renal biopsy (in two the HIV infection was first diagnosed at the time of renal biopsy).

Because of the significant relationship between HIV and renal AA-amyloidosis in this cohort we compared these data to those of 25 HIV-positive patients without a prior history of IVDU that underwent renal biopsy within the same time range, and found none of them having renal AA-amyloidosis (data not shown).

\section{Discussion}

In this retrospective study we found renal AAamyloidosis to be the predominant cause of progressive kidney disease in IVDU accounting for $50 \%$ of cases, followed by membranoproliferative glomerulonephritis as the second leading cause in $20.8 \%$. This finding is well in accordance with two recent observations from other European Centres. These studies described an increasing rate of renal AA-amyloidosis in IVDU, while in older biopsy studies AA-amyloidosis was found only sporadically, indicating a changing pattern of nephropathy in this patient population [5-7].

The occurrence of renal AA-amyloidosis in IVDU has been linked to repeated bacterial infections, especially to skin and soft tissue infections, endocarditis as well as longer survival time after the onset of IVDU (as a consequence of successful opoid maintenance therapy) $[6,7,10,11]$. When comparing patients with renal AAamyloidosis to those without, we found a trend towards a longer self-reported time of IVDU. Repeated infections tended also to be more frequent in patients with AAamyloidosis. Yet, the low number of patients in our series makes it difficult to establish a link between isolated sites of infection and occurrence of AA-amyloidosis. Alternatively, it might be the case that not one 


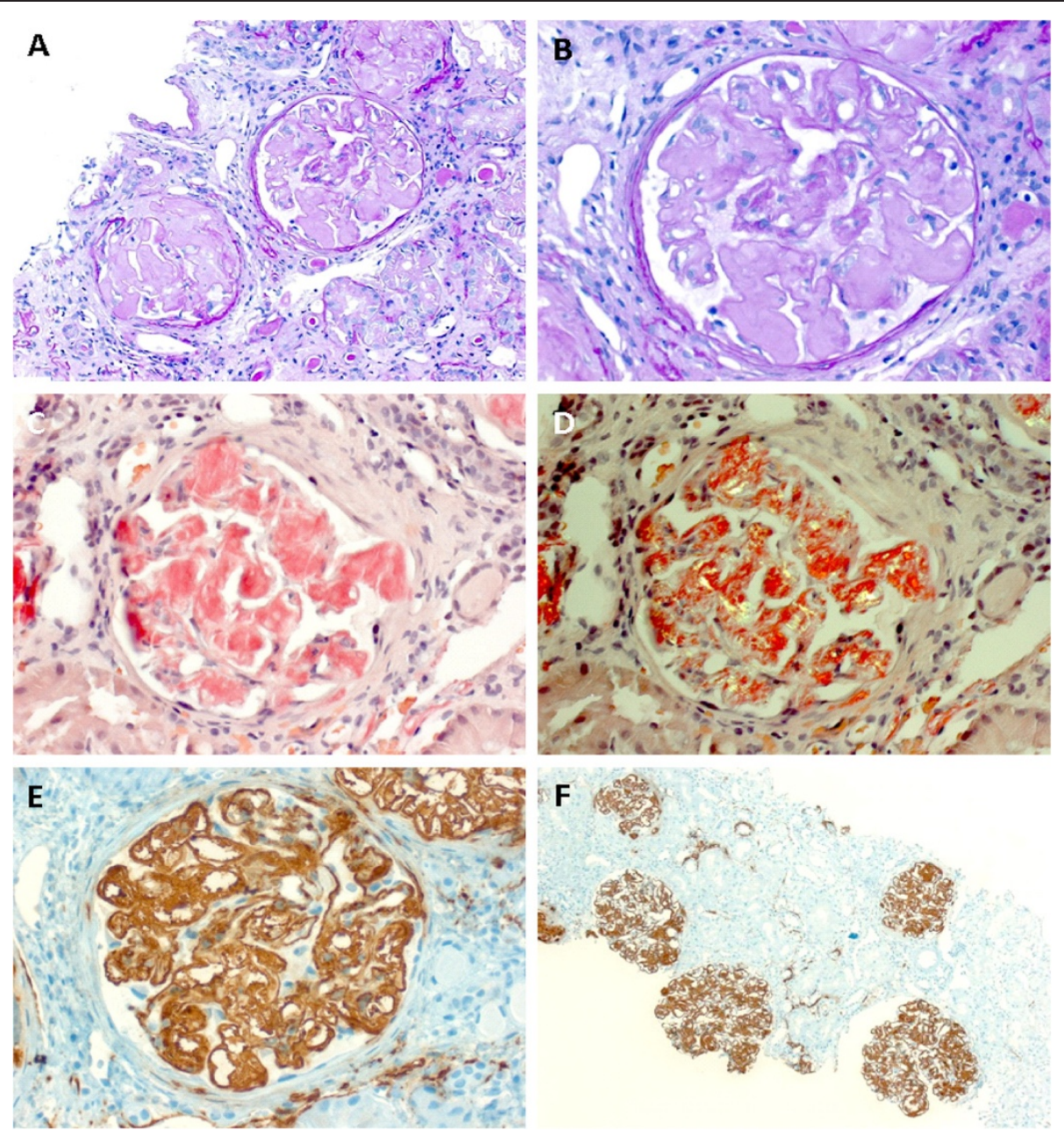

Figure 1 Representative histological findings in a case of renal AA-amyloidosis.

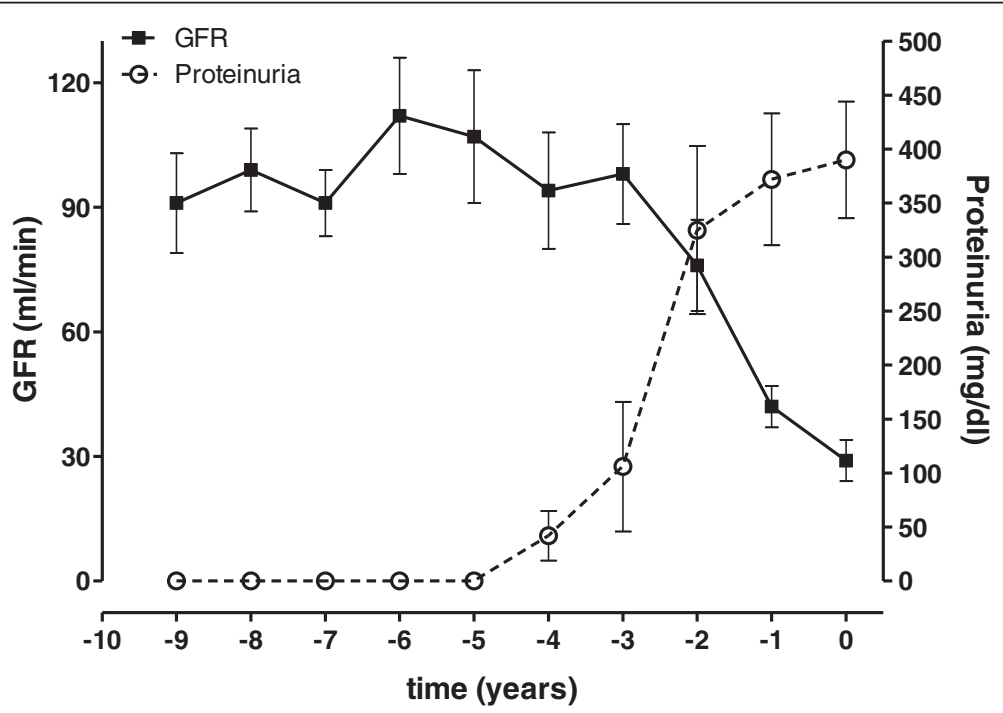

Figure 2 Clinical course of renal AA-amyloidosis prior to renal biopsy. 


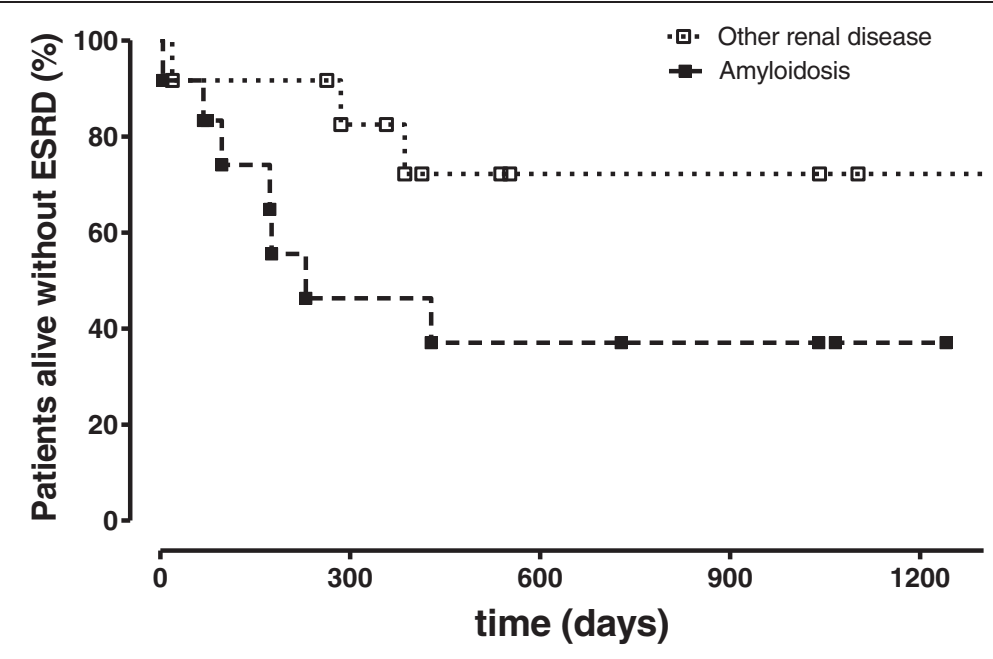

Figure 3 Outcome in patients with diagnosed renal AA-amyloidosis compared to those with other types of disease.

specific focus but the mere total number of bacterial infections predisposes to the development of renal amyloidosis.

The proportion of patients with HIV infection was significantly higher in patients with renal AA-amyloidosis. This observation has not been made in two published studies on AA-amyloidosis in patients with IVDU. One study did not provide data concerning HIV serostatus, while the other found only one out of 20 patients to be HIV infected [6,7]. However, this does not rule out a higher rate of HIV infections in these studies, as in some of our patients the HIV infection was just diagnosed before the renal biopsy was conducted. Several cases of amyloidosis in HIV infected IVDU have been described in the literature $[5,6,13,14,16,17]$. Besides IVDU, renal amyloidosis in HIV infected patients has been described in association with visceral leishmaniosis or multicentric Castleman`s disease [18-21], which were both not found in our cohort. During the same time period of this retrospective study, 25 HIV-infected patients without a

Table 2 Comparison of patient's characteristics

\begin{tabular}{|c|c|c|c|}
\hline & AA-Amyloidosis & Other renal & $p$-value \\
\hline & $(n=12)$ & $\overline{\text { disease }(n=12)}$ & \\
\hline Age (years) & $39.5(32-44)$ & $43.5(33-48)$ & 0.563 \\
\hline Male - n (\%) & $9(75.0 \%)$ & $6(50 \%)$ & 0.400 \\
\hline Self-reported time after initiation of IVDU (years) & $22(17-31)$ & $19.5(11-29)$ & 0.418 \\
\hline Self-reported duration of IVDU (years) & $20.5(14-27)$ & $12.5(7-19)$ & 0.056 \\
\hline History of opioid maintenance therapy & $5(41.7 \%)$ & $7(58.3 \%)$ & 0.684 \\
\hline Chronic hepatitis B & $1(4.2 \%)$ & $0(0 \%)$ & 1.0 \\
\hline Chronic hepatitis C & $10(83.3 \%)$ & $11(91.7 \%)$ & 1.0 \\
\hline HIV & $8(66.7 \%)$ & $2(16.7 \%)$ & 0.036 \\
\hline \multicolumn{4}{|l|}{ Medical history of } \\
\hline Chronic or repeated skin infections & $11(91.7 \%)$ & $6(50.0 \%)$ & 0.069 \\
\hline Bacterial endocarditis & $2(16.7 \%)$ & $1(8.3 \%)$ & 1.000 \\
\hline Repeated pneumonia & $8(66.7 \%)$ & $3(25.0 \%)$ & 0.100 \\
\hline Serum creatinine (mg/dl) & $2.5(1.9-4.0)$ & $2.4(1.7-3.2)$ & 0.902 \\
\hline Proteinuria (g/24 h) & $8.9(7.5-19.1)$ & $4.1(1.5-9.8)$ & 0.021 \\
\hline Serum albumin & $2.2(1.5-2.8)$ & $3.0(2.0-3.9)$ & 0.124 \\
\hline Total serum protein & $6.2(5.3-7.2)$ & $6.1(4.9-7.4)$ & 0.853 \\
\hline Peripheral edema & $3(25.0 \%)$ & $4(33.3 \%)$ & 1.0 \\
\hline Hypertension & $1(8.3 \%)$ & $5(60.0 \%)$ & 0.1 \\
\hline
\end{tabular}

Variables are expressed as median and interquartile-range (IQR) or as proportions as appropriate. 
history of IVDU underwent renal biopsy at our institutions and none of them had evidence of renal AAamyloidosis [data not shown]. Although these patients cannot be properly matched, because of a higher median age and 25\% being of African-descent, this could indicate that the HIV infection by itself does not predispose for AA-amyloidosis, but coeval IVDU might increase the risk. Indeed, in a recent study, we found IVDU to be an independent risk factor for ESRD in an HIV-infected population [22]. Moreover, another study demonstrated that the combination of HIV infection and IVDU increases the risk for chronic kidney disease (CKD) in IVDU, with a higher prevalence of proteinuria in HIVinfected compared to uninfected IVDU [23]. The majority of HIV infected patients with renal AA-amyloidosis had a poorly or uncontrolled HIV infection prompting a severe immunosuppression and a chronic inflammation. Thus the uncontrolled HIV infection might be an important direct and indirect factor for the development of AA-amyloidosis predisposing to more frequent and severe bacterial infections.

Although there are several case reports of AAamyloidosis in HIV infected IVDU, such a high rate has not been previously described. What might explain this finding? First, medical management of the IVDU population is challenging, as patients often do not return for follow-up and seek medical advice only when acutely ill. Hence, renal biopsy is infrequently performed in IVDU. Second, the time course of renal amyloidosis, its clinical presentation with lack of peripheral edema or systemic hypertension, and enlarged echogenic kidneys on ultrasound makes it indistinguishable from certain other types of renal disease, especially HIV-associated nephropathy (HIVAN) $[3,17,24]$. The diagnosis of HIVAN is often established solely clinically and is often not confirmed by renal biopsy. Thus the true prevalence of renal AA-amyloidosis might even be underestimated $[25,26]$. Third, development of AA-amyloidosis also depends on predisposing genetic factors and is more frequent in Caucasian compared to Black patients. Vice versa HIVAN occurs mostly in Black patients and is a rarity in Caucasians [27]. Thus different ethnic compositions of IVDU cohorts may also contribute to the higher incidence observed by us and other European centres when compared to studies from the US with far higher rates of black IVDU [6,7].

IVDU patients with AA-amyloidosis had a poor prognosis, when compared to patients with other underlying causes of AA-amyloidosis [6-8]. We and others found that patients, who remained abstinent from IVDU after the initial diagnosis, achieved stable or even improving renal function during follow-up. This supports the idea that removal of the recurrent inflammatory stimuli can lead to at least some reversal of disease [6]. These observations, underscore the importance of renal biopsies, especially in the light of the oligosymptomatic clinical presentation of these patients. We found that overt proteinuria preceded the GFR decline by approximately 1-2 years. But unfortunately urine analysis is often missed by IVDU because unannounced illicit drug testing with possible consequences for opioid maintenance therapy is feared.

Besides the already discussed, our study has several limitations. First, the population studied is small and from a single metropolitan area. Thus, our results cannot be generalized to other HIV, often ethnically highly divers, cohorts. Second, IVDU often consume more than one substance of varying, uncontrolled purity. Thus our retrospective study cannot determine possible differences between substances used.

\section{Conclusion}

In conclusion we found AA-amyloidosis was the predominant cause of progressive renal disease in the last 10 years in IVDU patients. The high incidence of AA-amyloidosis observed was related to coeval HIV infection. We speculate that the HIV mediated immunosuppression promotes development of AA-amyloidosis by increasing frequency and severity of infectious complications acquired by ongoing IVDU. Overt proteinuria preceded the decline of GFR for approximately $1-2$ years, providing an opportunity for early intervention in an otherwise fatal disease.

\section{Competing interests}

All authors declare that they have no competing interests and have had no involvements that might raise the question of bias in the work reported or in the conclusions, implications, or opinions stated. No funding was obtained for this study.

\section{Authors' contributions}

$\mathrm{OJ}$ and MB initiated the study, treated patients, collected and analyzed the data and wrote the manuscript. MBüttner and KA examined the renal biopsies. All other authors treated the patients and are substantially involved in the collection and monitoring of data. No funding was obtained for this study. All authors read and approved the final manuscript.

\section{Author details}

${ }^{1}$ Department of Nephrology, Goethe University, Frankfurt/Main, Germany. ${ }^{2}$ Department of Nephrology, Agaplesion Markus Krankenhaus, Frankfurt/Main, Germany. ${ }^{3}$ Institute of Pathology, Nephropathology, Friedrich-Alexander-University, Erlangen, Germany. ${ }^{4}$ Department of Infectious Disease, Goethe University, Frankfurt/Main, Germany. ${ }^{5}$ Klinikum der Goethe-Universität, Zentrum der Inneren Medizin II, Infektionsambulanz, Haus 68, 1 OG, Theodor Stern Kai 7, 60590, Frankfurt/Main, Germany

Received: 4 September 2012 Accepted: 18 November 2012 Published: 21 November 2012

\section{References}

1. Rao TK, Niccastri AD, Friedmann EA: Natural history of heroin associated nephropathy. N Engl J Med 1974, 290:19-23.

2. Kimmel PL: Chronic nephropathies of cocaine and heroin abuse: a critical review. Clin J Am Soc Nephrol 2006, 1:655-667.

3. Klotman PE: HIV-associated nephropathy. Kidney Int 1999, 55:1161-1176. 
4. Lucas GM, Mehta SH, Atta MG, et al: End-stage renal disease and chronic kidney disease in a cohort of African-American HIV-infected and at-risk HIV-seronegative participants followed between 1988 and 2004. AIDS 2007, 21:2435-2443.

5. Do Sameiro Faria M, Sampaio S, Faria V, Carvalho E: Nephropathy associated with heroin abuse in Caucasian patients. Nephrol Dial Transplant 2003, 18:2308-2313.

6. Connolly JO, Gillmore JD, Lachmann HJ, Davenport A, Hawkins PN, Woolfson RG: Renal amyloidosis in intravenous drug users. QJM 2006, 99:737-742.

7. Manner I, Sagedal S, Røger M, Os I: Renal amyloidosis in intravenous heroin addicts with nephrotic syndrome and renal failure. Clin Nephrol 2009, 72:224-228.

8. Lachmann HJ, Goodman HJ, Gilbertson JA, Gallimore JR, Sabin CA, Gillmore JD, Hawkins PN: Natural history and outcome in systemic AA amyloidosis. N Engl J Med 2007, 356:2361-2371.

9. Dember LM: Amyloidosis-associated kidney disease. J Am Soc Nephrol 2006, 17(12):3458-3471.

10. Menchel S, Cohen D, Gross E, Frangione B, Gallo G: AA protein-related renal amyloidosis in drug addicts. Am J Pathol 1983, 112:195-199.

11. Dubrow A, Mittman N, Ghali $V$, Flamenbaum W: The changing spectrum of heroin-associated nephropathy. Am J Kidney Dis 1985, 5:36-41.

12. Miranda BH, Connolly JO, Burns AP: Secondary amyloidosis in a needle phobic intra-venous drug user. Amyloid 2007, 14:255-258.

13. Newey C, Odedra BJ, Standish RA, Furmali R, Edwards SG, Miller RF: Renal and gastrointestinal amyloidosis in an HIV-infected injection drug user. Int J STD AIDS 2007, 18:357-358.

14. Chan-Tack KM, Ahuja N, Weinman EJ, Wali RK, Uche A, Greisman LA, Drachenberg C, Hawkins PN, Redfield RR: Acute renal failure and nephrotic range proteinuria due to amyloidosis in an HIV-infected patient. Am J Med Sci 2006, 332:364-367.

15. Levey AS, Bosch JP, Lewis JB, Greene T, Rogers N, Roth D: A more accurate method to estimate glomerular filtration rate from serum creatinine: a new prediction equation, Modification of Diet in Renal Disease Study Group. Ann Intern Med 1999, 130:461-470.

16. Nebuloni M, Barbiano Di Belgiojoso G, Genderini A, Tosoni A, Riani LN, Heidempergher M, Zerbi P, Vago L: Glomerular lesions in HIV-positive patients: a 20-year biopsy experience from Northern Italy. Clin Nephrol 2009, 72:38-45.

17. Atta MG, Choi MJ, Longenecker JC, Haymart M, Wu J, Nagajothi N, Racusen LC, Scheel PJ Jr, Brancati FL, Fine DM: Nephrotic range proteinuria and CD4 count as noninvasive indicators of HIV-associated nephropathy. Am J Med 2005, 118:1288.

18. El Karoui K, Vuiblet V, Dion D, Izzedine H, Guitard J, Frimat L, Delahousse M, Remy P, Boffa JJ, Pillebout E, Galicier L, Noël LH, Daugas E: Renal involvement in Castleman disease. Nephrol Dial Transplant 2011, 26:599-609.

19. de Vallière $S$, Mary $C$, Joneberg JE, Rotman S, Bullani R, Greub G, Gillmore JD, Buffet PA, Tarr PE: AA-amyloidosis caused by visceral leishmaniasis in a human immunodeficiency virus-infected patient. Am J Trop Med Hyg 2009, 81:209-212.

20. Navarro M, Bonet J, Bonal J, Romero R: Secondary amyloidosis with irreversible acute renal failure caused by visceral leishmaniasis in a patient with AIDS. Nefrologia 2006, 26:745-746. Spanish.

21. Amann K, Bogdan C, Harrer T, Rech J: Renal leishmaniasis as unusual cause of nephrotic syndrome in an HIV patient. J Am Soc Nephrol 2012, 23(4):586-590.

22. Bickel M, Stephan C, Nisius G, et al: ESRD and dialysis in HIV+ patientsobservations from a long-term cohort study with 22 years of follow-up. In Presented at the 19th Conference on Retroviruses and Opportunistic Infections (CROI), HIV Med.; 2012. doi:10.1111/j.1468-1293.2012.01045.x [Epub ahead of print].

23. Yanik EL, Lucas GM, Vlahov D, Kirk GD, Mehta SH: HIV and proteinuria in an injection drug user population. Clin J Am Soc Nephrol 2010, 5:1836-1843.

24. Weiner NJ, Goodman JW, Kimmel PL: The HIV-associated renal diseases: current insight into pathogenesis and treatment. Kidney Int 2003, 63:1618-1631.

25. Rodriguez RA, Mendelson M, O'Hare AM, Hsu LC, Schoenfeld P: Determinants of survival among HIV-infected chronic dialysis patients. J Am Soc Nephrol 2003, 14:1307-1313.
26. Post FA, Campbell LJ, Hamzah L, Collins L, Jones R, Siwani R, Johnson L, Fisher M, Holt SG, Bhagani S, Frankel AH, Wilkins E, Ainsworth JG, Larbalestier N, Macallan DC, Banerjee D, Baily G, Thuraisingham RC, Donohoe P, Hendry BM, Hilton RM, Edwards SG, Hangartner R, Howie AJ, Connolly JO, Easterbrook PJ: Predictors of renal outcome in HIV-associated nephropathy. Clin Infect Dis 2008, 46:1282-1289.

27. Obici L, Raimondi S, Lavatelli F, Bellotti V, Merlini G: Susceptibility to AA amyloidosis in rheumatic diseases: a critical overview. Arthritis Rheum 2009, 61:1435-1440. Review.

doi:10.1186/1471-2369-13-151

Cite this article as: Jung et al:: Renal AA-amyloidosis in intravenous drug users - a role for HIV-infection? BMC Nephrology 2012 13:151.

\section{Submit your next manuscript to BioMed Central and take full advantage of:}

- Convenient online submission

- Thorough peer review

- No space constraints or color figure charges

- Immediate publication on acceptance

- Inclusion in PubMed, CAS, Scopus and Google Scholar

- Research which is freely available for redistribution 